\title{
ITERATIVE ALGORITHMS BASED ON DECOUPLING OF DEBLURRING AND DENOISING FOR IMAGE RESTORATION*
}

\author{
YOU-WEI WEN ${ }^{\dagger}$, MICHAEL K. NG ${ }^{\ddagger}$, AND WAI-KI CHING ${ }^{\S}$
}

\begin{abstract}
In this paper, we propose iterative algorithms for solving image restoration problems. The iterative algorithms are based on decoupling of deblurring and denoising steps in the restoration process. In the deblurring step, an efficient deblurring method using fast transforms can be employed. In the denoising step, effective methods such as the wavelet shrinkage denoising method or the total variation denoising method can be used. The main advantage of this proposal is that the resulting algorithms can be very efficient and can produce better restored images in visual quality and signalto-noise ratio than those by the restoration methods using the combination of a data-fitting term and a regularization term. The convergence of the proposed algorithms is shown in the paper. Numerical examples are also given to demonstrate the effectiveness of these algorithms.
\end{abstract} ation

Key words. iterative algorithms, image restoration, deblurring, denoising, wavelet, total vari-

AMS subject classifications. 65F10,65F22, 65K10

DOI. $10.1137 / 070683374$

1. Introduction. Digital image restoration and reconstruction play an important part in various areas of applied sciences such as medical and astronomical imaging, film restoration, and image and video coding. In this paper, we focus on a common degradation model [17]: an ideal image $\boldsymbol{f} \in \mathbb{R}^{N}$ is observed in the presence of a Toeplitz matrix $\boldsymbol{H} \in \mathbb{R}^{N \times N}$ arising from a spatial-invariant blur and an additive zero-mean Gaussian white noise $\boldsymbol{n} \in \mathbb{R}^{N}$ of standard deviation $\sigma$. Thus the observed image $\boldsymbol{g} \in \mathbb{R}^{N}$ is obtained by

$$
g=H f+n .
$$

It is well known that restoring an image $f$ is a very ill-conditioned problem. A regularization method should be used in the image restoration process. One usual approach is to determine the restored image by minimizing a cost function consisting of a data-fitting term and a regularization term:

$$
\min _{\boldsymbol{f}}\|\boldsymbol{H} \boldsymbol{f}-\boldsymbol{g}\|_{2}^{2}+\alpha \mathcal{R}(\boldsymbol{f}) .
$$

Here $\|\cdot\|_{2}$ is the Euclidean norm in $\mathbb{R}^{N}, \mathcal{R}(\boldsymbol{f})$ is the regularization term, and $\alpha$ is a positive regularization parameter. Numerous expressions for $\mathcal{R}$ have been used in the literature:

* Received by the editors February 21, 2007; accepted for publication (in revised form) March 21, 2008; published electronically August 6, 2008.

http://www.siam.org/journals/sisc/30-5/68337.html

†Faculty of Science, South China Agricultural University, Wushan, Guangzhou 510642, People’s Republic of China (wenyouwei@graduate.hku.hk). This author's research was supported in part by NNSFC grant 60702030 .

$\ddagger$ Corresponding author. Centre for Mathematical Imaging and Vision and Department of Mathematics, Hong Kong Baptist University, Kowloon Tong, Hong Kong (mng@math.hkbu.edu.hk). This author's research was supported in part by RGC grants 7045/04P and 7045/05P and HKBU FRGs.

$\S$ Department of Mathematics, The University of Hong Kong, Pokfulam Road, Hong Kong (wching@hkusua.hku.hk). 
- Tikhonov regularization $[15,32]: \mathcal{R}(\boldsymbol{f})=\|\boldsymbol{f}\|_{2}^{2}$ or $\|\nabla \boldsymbol{f}\|_{2}^{2}$, where $\nabla$ stands for the discrete gradient. It has been shown in [25] that an efficient image restoration method based on fast transforms can be developed, and the computational cost is $O(N \log N)$ operations. However, the drawback of the Tikhonov regularization is that image edges cannot be preserved in the restoration process.

- Total variation (TV) regularization [29]: $\mathcal{R}(\boldsymbol{f})=\|\nabla \boldsymbol{f}\|_{2}$. The distinctive feature of TV regularization is that image edges can be preserved. Thus TV regularization is in general more suitable than the Tikhonov regularization for image restoration purposes. We refer readers to [28] for recent developments in TV image restoration.

- Wavelet regularization $[2,10,13]: \mathcal{R}(\boldsymbol{f})=\sum_{k} \lambda_{k} \phi\left(\left\langle\boldsymbol{f}, \boldsymbol{\psi}_{k}\right\rangle\right)$, where $\phi(\cdot)$ is a penalty function, $\lambda_{k}$ denotes a weighted coefficient, and $\left\{\boldsymbol{\psi}_{k}\right\}$ denotes a wavelet orthonormal basis. In image restoration, $\phi(x)=|x|^{p}$, where $1 \leq p \leq 2$ is commonly used.

In this paper, we propose iterative algorithms for solving image restoration problems. The iterative algorithms are based on decoupling of deblurring and denoising steps in the image restoration process. The motivations of this approach are that

(M1) an efficient deblurring method based on fast transforms can be used in the deblurring step;

(M2) effective denoising methods such as the wavelet shrinkage method and the TV method can be employed in the denoising step;

(M3) the resulting algorithms can be very efficient and can produce better restored images than those of the restoration method using data-fitting and regularization terms together in (1.2).

We consider and study the following iterative algorithms based on decoupling of deblurring and denoising steps (an initial guess $\boldsymbol{f}^{(0)}$ is used).

Algorithm 1.

$$
\begin{aligned}
\hat{\boldsymbol{f}}^{(i)} & =\underset{\boldsymbol{f}}{\operatorname{argmin}}\|\boldsymbol{H} \boldsymbol{f}-\boldsymbol{g}\|_{2}^{2}+\alpha_{1}\left\|\boldsymbol{f}-\boldsymbol{f}^{(i-1)}\right\|_{2}^{2}, \\
\boldsymbol{f}^{(i)} & =\underset{\boldsymbol{f}}{\operatorname{argmin}}\left\|\boldsymbol{f}-\hat{\boldsymbol{f}}^{(i)}\right\|_{2}^{2}+\sum_{k} \lambda_{k} \phi\left(\left\langle\boldsymbol{f}, \boldsymbol{\psi}_{k}\right\rangle\right) .
\end{aligned}
$$

\section{Algorithm 2.}

$$
\begin{aligned}
& \hat{\boldsymbol{f}}^{(i)}=\underset{\boldsymbol{f}}{\operatorname{argmin}}\|\boldsymbol{H} \boldsymbol{f}-\boldsymbol{g}\|_{2}^{2}+\alpha_{1}\left\|\boldsymbol{f}-\boldsymbol{f}^{(i-1)}\right\|_{2}^{2}, \\
& \boldsymbol{f}^{(i)}=\underset{\boldsymbol{f}}{\operatorname{argmin}}\left\|\boldsymbol{f}-\hat{\boldsymbol{f}}^{(i)}\right\|_{2}^{2}+\alpha_{2} T V(\boldsymbol{f}) .
\end{aligned}
$$

We can also apply the wavelet denoising method and then the TV denoising method in the denoising step. The main reason is that Gibbs-type oscillation artifacts may be introduced in the restored signal by using wavelet denoising methods; see, for instance, Figure 3(b) in the next subsection. When the TV denoising method is used after the wavelet denoising method, oscillation artifacts can be suppressed; see, for instance, Figure 3(d) in the next subsection. 


\section{Algorithm 3.}

$$
\begin{aligned}
\hat{\boldsymbol{f}}^{(i)} & =\underset{\boldsymbol{f}}{\operatorname{argmin}}\|\boldsymbol{H} \boldsymbol{f}-\boldsymbol{g}\|_{2}^{2}+\alpha_{1}\left\|\boldsymbol{f}-\boldsymbol{f}^{(i-1)}\right\|_{2}^{2}, \\
\tilde{\boldsymbol{f}}^{(i)} & =\underset{\boldsymbol{f}}{\operatorname{argmin}}\left\|\boldsymbol{f}-\hat{\boldsymbol{f}}^{(i)}\right\|_{2}^{2}+\sum_{k} \lambda_{k} \phi\left(\left\langle\boldsymbol{f}, \boldsymbol{\psi}_{k}\right\rangle\right), \\
\boldsymbol{f}^{(i)} & =\underset{\boldsymbol{f}}{\operatorname{argmin}}\left\|\boldsymbol{f}-\tilde{\boldsymbol{f}}^{(i)}\right\|_{2}^{2}+\alpha_{2} T V(\boldsymbol{f}) .
\end{aligned}
$$

In the above three algorithms, $\alpha_{1}$ and $\alpha_{2}$ are the positive parameters for deblurring and denoising, respectively.

We note that the computational cost of the deblurring step in (1.3), (1.5), and (1.7) is $O(N \log N)$ operations [25], and both wavelet denoising methods [11, 16, 22] and the TV denoising method $[5,6,26]$ in the denoising steps are also very efficient. These refer to our motivations (M1) and (M3). In the next subsection, we consider a one-dimensional signal restoration example to further illustrate our motivations (M2) and (M3).

We remark that we use wavelet shrinkage and/or total variation in the denoising step. However, the other effective denoising methods can be used in the framework if they are available and suitable for the purpose.

1.1. An example. The original signal is shown in Figure 1 . The following experiments are studied:

(i) The original signal is blurred by a motion blur of length 9 and degraded by the Gaussian noise with a zero mean and a variance of 64 . The blurred and noisy signal is then shown in Figure 2(a). Figures 2(b) and 2(c) show the restored signals by minimizing a cost function (cf. (1.2)) consisting of a data-fitting term and a regularization term of the Haar wavelet (hard thresholding) regularization method [13] and of the TV regularization method [29], respectively. Here trial by error for different values of $\alpha$ in (1.2) is used. In the figures, the least relative errors of the restored signals among the tested values of $\alpha$ are reported.

(ii) The original signal is degraded only by the Gaussian noise with a zero mean and a variance of 64 . The noisy signal is then shown in Figure 3(a). Figures 3(b) and 3(c) show the restored signals by minimizing a cost function

$$
\min _{\boldsymbol{f}}\|\boldsymbol{f}-\boldsymbol{g}\|_{2}^{2}+\alpha \mathcal{R}(\boldsymbol{f})
$$

$(\boldsymbol{H}=\boldsymbol{I}$, i.e., no blur $)$ using the Haar wavelet hard thresholding denoising method and the TV denoising method, respectively. We observe that the Haar wavelet hard thresholding method [22] (Figure 3(b)) and the TV method (Figure 3(c)) are more effective in denoising (both methods have smaller relative errors) than in the combination of deblurring and denoising together (Figures 2(b)-(c)). This illustrates our motivation (M2).

(iii) For the motivation (M3), we used the three proposed iterative algorithms to restore the blurred and noisy signal in Figure 2(a). Figures 4(a), 4(b), and 4(c) show the restored signals by using Algorithms 1, 2, and 3, respectively. In these tests, the Haar wavelet hard thresholding denoising method [22] is used. We see from the figures that the relative errors of the restored signals by Algorithms 1 and 2 are less than those of Figures 2(b) and 2(c). We also find that the relative errors of the restored signals by Algorithm 3 are the 


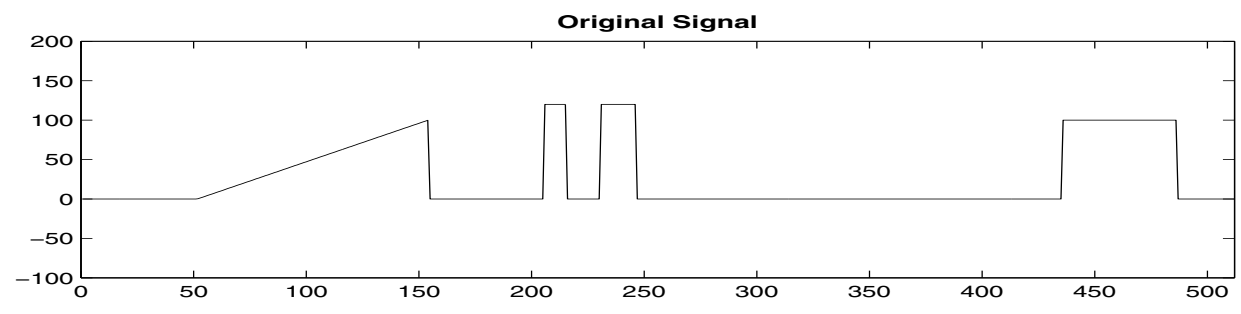

FIG. 1. The original signal.

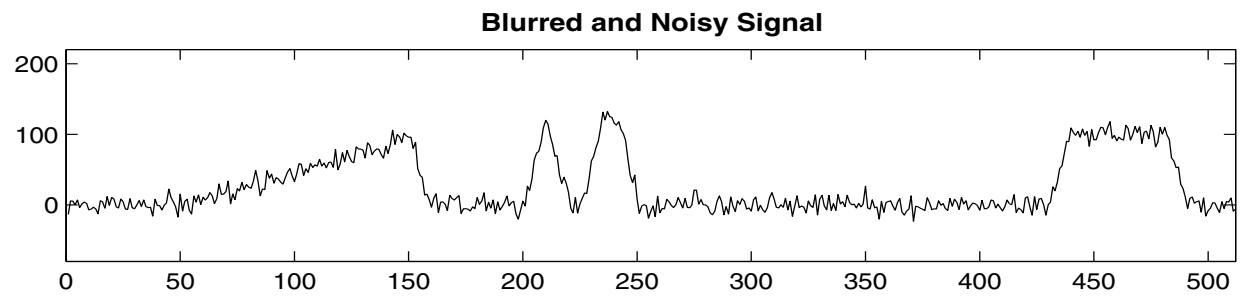

(a)

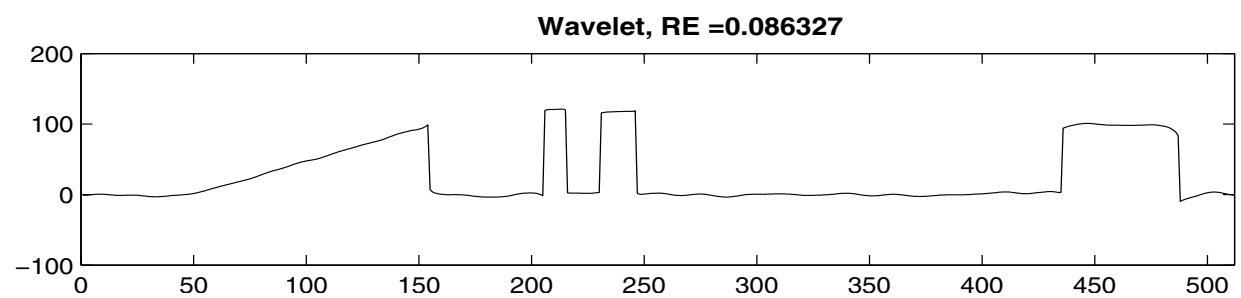

(b)

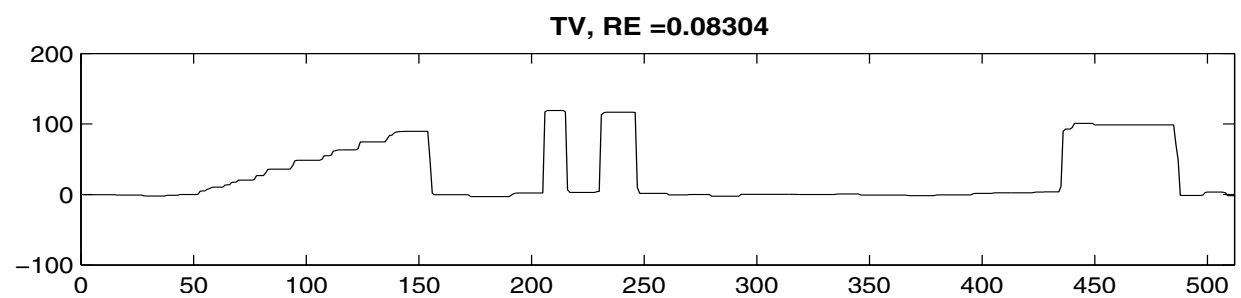

(c)

FIG. 2. The blurred and noisy signal (a); the restored signal by the Haar wavelet regularization method (b); the restored signal by the TV regularization method (c).

least among all the restored signals. These examples show that decoupling of deblurring and denoising for image restoration can be an effective approach. In section 4, more numerical examples will be given to demonstrate the effectiveness of this approach.

1.2. Outline. The outline of this paper is as follows. In section 2 , we review deblurring and denoising methods. In section 3, we show the convergence of Algorithms 1,2 , and 3 . In section 4 , numerical examples are presented to demonstrate the effectiveness of the proposed methods. Finally, concluding remarks are given in section 5 . 


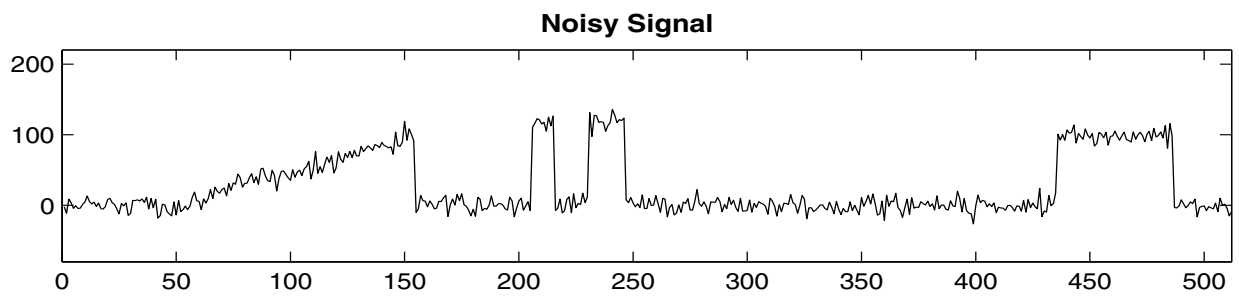

(a)

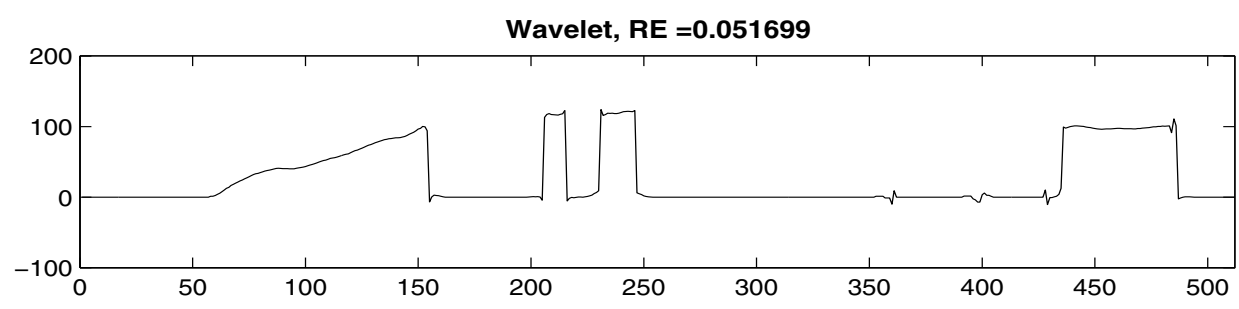

(b)
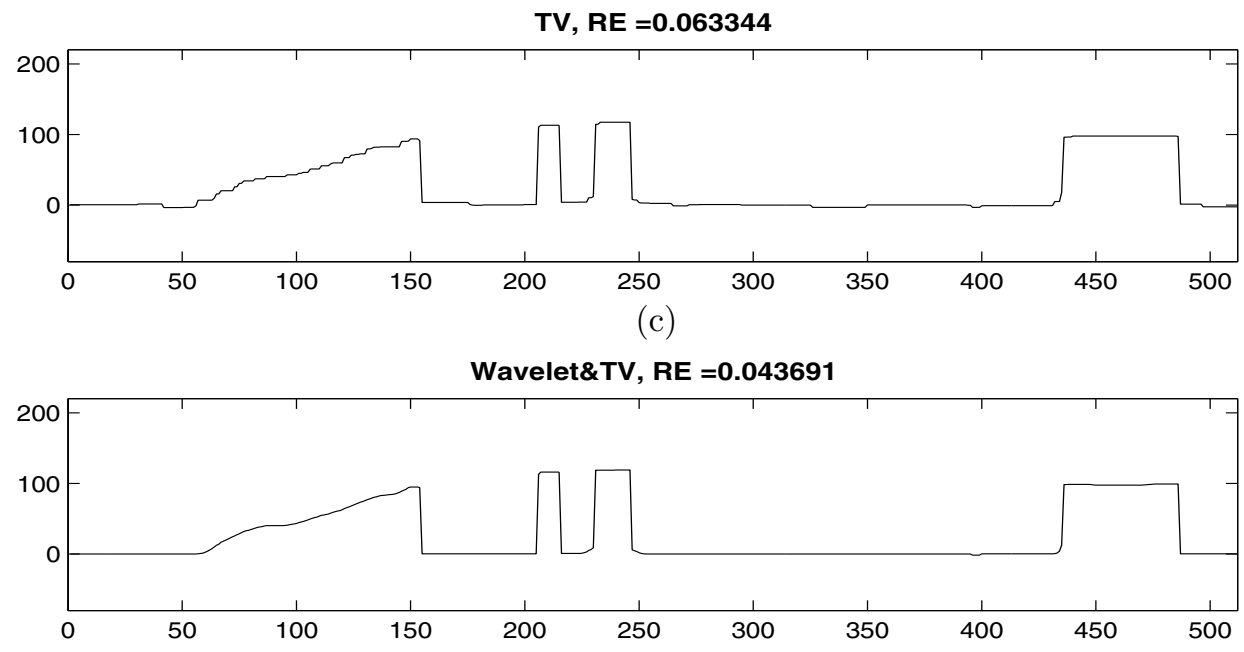

(d)

FIG. 3. The noisy signal (a); the restored signal by the Haar wavelet regularization method (b); the restored signal by the TV regularization method (c); the restored signal by the Haar wavelet regularization method and then the $T V$ regularization method (d).

2. Deblurring and denoising methods. In this section, we review deblurring and denoising methods.

2.1. Deblurring methods. In order to determine $\hat{\boldsymbol{f}}^{(i)}$ in (1.3), (1.5), and (1.7), it is required to solve the following linear system:

$$
\left(\boldsymbol{H}^{T} \boldsymbol{H}+\alpha_{1} \boldsymbol{I}\right) \hat{\boldsymbol{f}}^{(i)}=\boldsymbol{H}^{T} \boldsymbol{g}+\alpha_{1} \boldsymbol{f}^{(i-1)} .
$$

Because of the regularized term $\alpha_{1} \boldsymbol{I}$, the coefficient matrix $\left(\boldsymbol{H}^{T} \boldsymbol{H}+\alpha_{1} \boldsymbol{I}\right)$ is always invertible even if $\boldsymbol{H}^{T} \boldsymbol{H}$ is singular.

We note that $\boldsymbol{H}$ is a block-circulant with circulant-block (BCCB) matrix when periodic boundary conditions are applied to the image boundary. The matrix $\boldsymbol{H}$ can 
Our Model (Wavelet), RE $=0.070538$

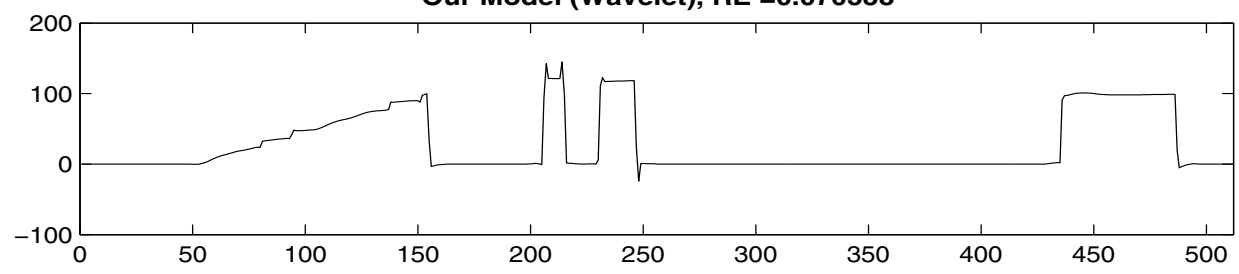

(a)

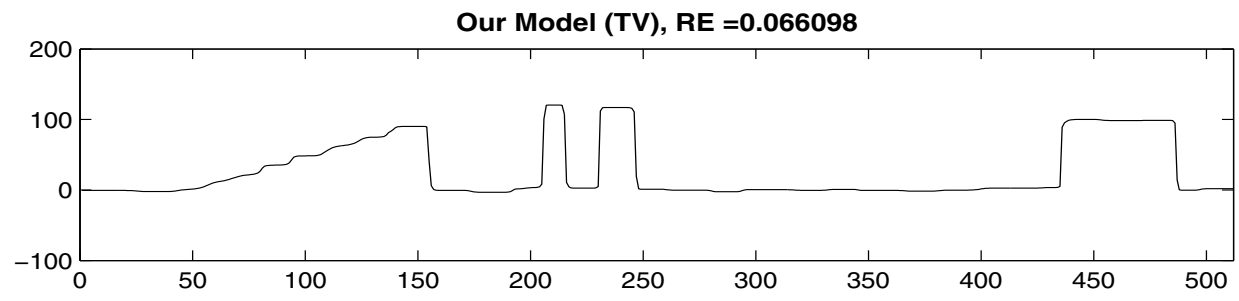

(b)

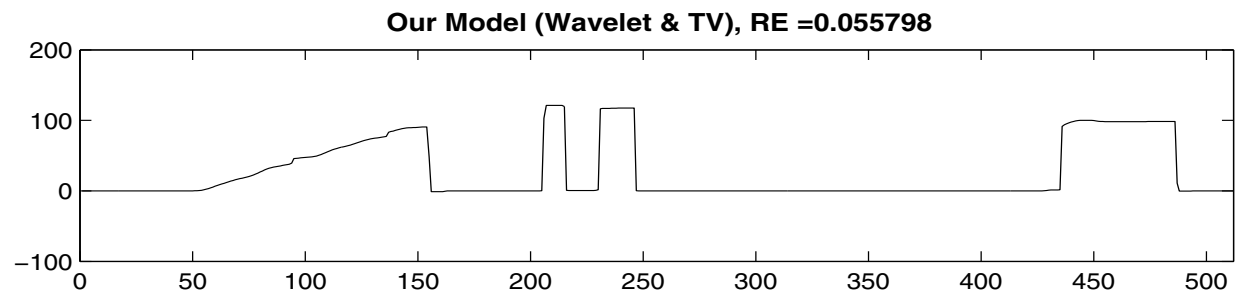

(c)

FIG. 4. The restored signal by Algorithm 1 (a); the restored signal by the TV regularization method and the restored signal by Algorithm 2 (b); the restored signal by Algorithm 3 (c).

be diagonalized by the discrete fast Fourier transform (FFT) matrix, and therefore the cost of solving (2.1) is $O(N \log N)$ operations, where $N$ is the number of pixels of the restored image. When zero boundary conditions are applied to the image boundary, $\boldsymbol{H}$ is a block-Toeplitz with Toeplitz-block (BTTB) matrix. The conjugate gradient method can be used to solve (2.1). The convergence of the conjugate gradient method can be improved by using preconditioning techniques. Transformation-based preconditioning techniques have proved to be very successful [24]. When reflective boundary conditions are applied to the image boundary, $\boldsymbol{H}$ is a block-Toeplitz-plusHankel with Toeplitz-plus-Hankel-block (BTHTHB) matrix. If the blurring function is symmetric, such a BTHTHB matrix can be diagonalized by the discrete cosine transform (DCT) matrix [25]. Therefore the cost of solving the corresponding linear system is $O(N \log N)$ operations.

We note from (1.1) and (2.1) that

$$
\hat{\boldsymbol{f}}^{(i)}=\left(\boldsymbol{H}^{T} \boldsymbol{H}+\alpha_{1} \boldsymbol{I}\right)^{-1}\left(\boldsymbol{H}^{T} \boldsymbol{g}+\alpha_{1} \boldsymbol{f}^{(i-1)}\right)=\boldsymbol{f}+\boldsymbol{\epsilon}_{f}^{(i)}+\boldsymbol{\epsilon}_{n},
$$

where

$$
\boldsymbol{\epsilon}_{f}^{(i)}=\alpha_{1}\left(\boldsymbol{H}^{T} \boldsymbol{H}+\alpha_{1} \boldsymbol{I}\right)^{-1}\left(\boldsymbol{f}^{(i-1)}-\boldsymbol{f}\right) \quad \text { and } \quad \boldsymbol{\epsilon}_{n}=\left(\boldsymbol{H}^{T} \boldsymbol{H}+\alpha_{1} \boldsymbol{I}\right)^{-1} \boldsymbol{H}^{T} \boldsymbol{n}
$$

Here the variance of $\boldsymbol{\epsilon}_{n}$ is given by $\sigma^{2}\left(\boldsymbol{H}^{T} \boldsymbol{H}+\alpha_{1} \boldsymbol{I}\right)^{-1} \boldsymbol{H}^{T} \boldsymbol{H}\left(\boldsymbol{H}^{T} \boldsymbol{H}+\alpha_{1} \boldsymbol{I}\right)^{-1}$. Ac- 
cording to (2.2), we will choose a small value of $\alpha_{1}$ in order to reduce the error $\boldsymbol{\epsilon}_{f}^{(i)}$. However, the residual noise $\boldsymbol{\epsilon}_{n}$ will be amplified by using a small value of $\alpha_{1}$. In the denoising step of Algorithms 1, 2, and 3, we apply wavelet shrinkage and/or TV regularization denoising methods to suppress the residual noise.

2.2. Wavelet denoising methods. In [34], Weaver et al. first proposed using wavelet shrinkage for filtering noise from images. Also wavelet-based noise reduction algorithms $[11,12,22]$ are asymptotically near-optimal for a wide class of signals corrupted by additive Gaussian white noises. These algorithms also work well when the noise is neither white noise nor Gaussian; see, for instance, [18].

By using the unitary invariance property of the 2-norm, one can rewrite

$$
\left\|\boldsymbol{f}-\hat{\boldsymbol{f}}^{(i)}\right\|_{2}^{2}=\sum_{k}\left(\left\langle\boldsymbol{f}, \boldsymbol{\psi}_{k}\right\rangle-\left\langle\hat{\boldsymbol{f}}^{(i)}, \boldsymbol{\psi}_{k}\right\rangle\right)^{2}
$$

in (1.4), (1.6), and (1.8). Therefore we have

$$
\boldsymbol{f}^{(i)}=\underset{\boldsymbol{f}}{\operatorname{argmin}} \sum_{k}\left(\left(\left\langle\hat{\boldsymbol{f}}^{(i)}, \boldsymbol{\psi}_{k}\right\rangle-\left\langle\boldsymbol{f}, \boldsymbol{\psi}_{k}\right\rangle\right)^{2}+\lambda_{k}\left|\left\langle\boldsymbol{f}, \boldsymbol{\psi}_{k}\right\rangle\right|^{p}\right) .
$$

It is easy to see that the wavelet coefficients of $\boldsymbol{f}^{(i)}$ can be obtained independently for each $\left\langle\boldsymbol{f}^{(i)}, \boldsymbol{\psi}_{k}\right\rangle$ as a scalar optimization problem. In particular, when $p=1$, the solution in (2.3) is just the popular soft thresholding scheme [12]:

$$
\left\langle\hat{\boldsymbol{f}}^{(i)}, \boldsymbol{\psi}_{k}\right\rangle= \begin{cases}\left\langle\hat{\boldsymbol{f}}^{(i)}, \boldsymbol{\psi}_{k}\right\rangle-\lambda_{k} / 2 & \text { if }\left\langle\hat{\boldsymbol{f}}^{(i)}, \boldsymbol{\psi}_{k}\right\rangle \geq \lambda_{k} / 2, \\ \left\langle\hat{\boldsymbol{f}}^{(i)}, \boldsymbol{\psi}_{k}\right\rangle+\lambda_{k} / 2 & \text { if }\left\langle\hat{\boldsymbol{f}}^{(i)}, \boldsymbol{\psi}_{k}\right\rangle \leq-\lambda_{k} / 2 \\ 0 & \text { otherwise }\end{cases}
$$

Usually, $\lambda_{k}$ is set to be a constant related to the variance $\sigma_{k}$ of the residual noise $\boldsymbol{\epsilon}_{n}$ in its $k$ th level wavelet decomposition; see [11]. In our setting, the variance $\sigma_{k}$ can be estimated as follows:

$$
\sigma_{k}^{2}=\mathbf{E}\left\{\left|\left\langle\boldsymbol{\epsilon}_{n}, \boldsymbol{\psi}_{k}\right\rangle\right|^{2}\right\}=\sum_{u, v} \frac{\sigma^{2}|H(u, v)|^{2}|\Psi(k, u, v)|^{2}}{\left(|H(u, v)|^{2}+\alpha_{1}\right)^{2}},
$$

where $\mathbf{E}(\cdot)$ is the expectation operator, and $H(u, v)$ and $\Psi(k, u, v)$ are the discrete Fourier transforms of $\boldsymbol{H}$ and $\boldsymbol{\psi}_{k}$, respectively. Both $H(u, v)$ and $\Psi(k, u, v)$ can be computed by using the FFT in $O(N \log N)$ operations. This optimization step can be done independently for each wavelet coefficient, and therefore the cost of wavelet denoising the image solution is in $O(N \log N)$ operations. Other wavelet shrinkage denoising schemes such as the hard thresholding method [22] and the garrote thresholding method [16] can be used in the denoising step of the proposed algorithms.

2.3. The TV denoising method. The use of TV regularization for image processing has been proposed by Rudin, Osher, and Fatemi [29]. The distinctive feature of TV regularization is that edges can be preserved. We remark that Steidl et al. [30] have shown the equivalence of soft wavelet shrinkage, TV diffusion, and TV regularization. Recent developments in TV image restoration can be found in [28].

The Euler-Lagrange equation for the TV denoising problem is given by the following nonlinear system of equations. The artificial time marching scheme proposed in Rudin, Osher, and Fatemi [29] obtains the solution of this nonlinear system of 
equations as the steady state of a parabolic partial differential equation. Indeed, it is equivalent to employing the following gradient descent method to solve the minimization problem. The drawback of this method is that the convergence rate of explicit methods can be very slow due to stability constraints. Implicit methods can be applied, but then one has to deal with the nonlinearity problem and the solution of the resulting linear systems. In [33], the lagged diffusivity fixed point iteration is introduced. This method consists of linearizing the nonlinear differential term in the nonlinear system of equations by lagging the diffusion coefficient one iteration behind. The resulting linear system can be solved efficiently by multigrid methods [33]. The algorithm can be interpreted in the framework of generalized Weiszfeld methods; see, for instance, [7]. As proved in [7], this method is monotonically convergent, in the sense that the objective function values evaluated at the iterates form a monotonically decreasing sequence, and the convergence rate is linear. In practice, this method is very robust. Recently, Chan and Chen [6] proposed a very fast multilevel method using primal relaxation for TV denoising problems. Chambolle [5] considered a dual formulation of the TV denoising problem and used the projected gradient method to solve the resulting constrained optimization problem. Based on the theory on semismooth operators, Ng et al. [26] studied semismooth Newton methods for computing the nonlinear projection. The convergence and numerical results have shown that the proposed algorithm is quite effective.

3. Convergence analysis. Let us first introduce the following notation in the subsequent discussion:

$$
\begin{gathered}
\boldsymbol{S}_{h}(\boldsymbol{x}) \equiv \underset{\boldsymbol{f}}{\operatorname{argmin}}\|\boldsymbol{H} \boldsymbol{f}-\boldsymbol{g}\|_{2}^{2}+\alpha_{1}\|\boldsymbol{f}-\boldsymbol{x}\|_{2}^{2}, \\
\boldsymbol{S}_{w}(\boldsymbol{x}) \equiv \underset{\boldsymbol{f}}{\operatorname{argmin}}\|\boldsymbol{f}-\boldsymbol{x}\|_{2}^{2}+\sum_{k} \lambda_{k} \phi\left(\left\langle\boldsymbol{f}, \boldsymbol{\psi}_{k}\right\rangle\right), \\
\boldsymbol{S}_{t v}(\boldsymbol{x}) \equiv \underset{\boldsymbol{f}}{\operatorname{argmin}}\|\boldsymbol{f}-\boldsymbol{x}\|_{2}^{2}+\alpha_{2} T V(\boldsymbol{f}) .
\end{gathered}
$$

The main aim of this section is to show the convergence of Algorithm 1,

$$
\boldsymbol{f}^{(i)}=\boldsymbol{S}_{w}\left(\boldsymbol{S}_{h}\left(\boldsymbol{f}^{(i-1)}\right)\right),
$$

Algorithm 2,

$$
\boldsymbol{f}^{(i)}=\boldsymbol{S}_{t v}\left(\boldsymbol{S}_{h}\left(\boldsymbol{f}^{(i-1)}\right)\right)
$$

and Algorithm 3,

$$
\boldsymbol{f}^{(i)}=\boldsymbol{S}_{t v}\left(\boldsymbol{S}_{w}\left(\boldsymbol{S}_{h}\left(\boldsymbol{f}^{(i-1)}\right)\right)\right)
$$

for $i=1,2, \ldots$. We will use the techniques of nonexpansive operators to show the convergence. Let us first introduce some definitions.

Definition 3.1 (see [8]). An operator $\boldsymbol{P}: \mathbb{R}^{N} \rightarrow \mathbb{R}^{N}$ is called nonexpansive if, for any $\boldsymbol{x}_{1}, \boldsymbol{x}_{2} \in \mathbb{R}^{N}$, we have

$$
\left\|\boldsymbol{P}\left(\boldsymbol{x}_{1}\right)-\boldsymbol{P}\left(\boldsymbol{x}_{2}\right)\right\|_{2} \leq\left\|\boldsymbol{x}_{1}-\boldsymbol{x}_{2}\right\|_{2} .
$$


If there exist a number $\beta \in(0,1)$ and a nonexpansive operator $\boldsymbol{T}: \mathbb{R}^{N} \rightarrow \mathbb{R}^{N}$ such that $\boldsymbol{P}=(1-\beta) \boldsymbol{I}+\beta \boldsymbol{T}$ is nonexpansive, then $\boldsymbol{P}$ is called $\beta$-averaged. In particular, when $\beta=1 / 2, \boldsymbol{P}$ is called a firmly nonexpansive operator.

We remark that an equivalent form of a firmly nonexpansive operator is defined as

$$
\left\|\boldsymbol{P}\left(\boldsymbol{x}_{1}\right)-\boldsymbol{P}\left(\boldsymbol{x}_{2}\right)\right\|_{2}^{2} \leq\left[\boldsymbol{P}\left(\boldsymbol{x}_{1}\right)-\boldsymbol{P}\left(\boldsymbol{x}_{2}\right)\right]^{T}\left(\boldsymbol{x}_{1}-\boldsymbol{x}_{2}\right) ;
$$

see, for instance, [9]. In fact, let $\boldsymbol{T}$ be a nonexpansive operator such that $\boldsymbol{P}=\frac{1}{2} \boldsymbol{I}+\frac{1}{2} \boldsymbol{T}$. For any $\boldsymbol{x}, \boldsymbol{y} \in \mathbb{R}^{N}$, we have

$$
\|\boldsymbol{T}(\boldsymbol{x})-\boldsymbol{T}(\boldsymbol{y})\|_{2}^{2}=4\|\boldsymbol{P}(\boldsymbol{x})-\boldsymbol{P}(\boldsymbol{y})\|_{2}^{2}+\|\boldsymbol{x}-\boldsymbol{y}\|_{2}^{2}-4(\boldsymbol{P}(\boldsymbol{x})-\boldsymbol{P}(\boldsymbol{y}))^{T}(\boldsymbol{x}-\boldsymbol{y}) .
$$

Since $\boldsymbol{T}$ is nonexpansive, i.e.,

$$
\|\boldsymbol{T}(\boldsymbol{x})-\boldsymbol{T}(\boldsymbol{y})\|_{2}^{2} \leq\|\boldsymbol{x}-\boldsymbol{y}\|_{2}^{2},
$$

we obtain the inequality (3.1). On the other hand, given an operator $\boldsymbol{P}$ with (3.1), we have

$$
\|(2 \boldsymbol{P}-\boldsymbol{I})(x)-(2 \boldsymbol{P}-\boldsymbol{I})(y)\|_{2}^{2} \leq\|\boldsymbol{x}-\boldsymbol{y}\|_{2}^{2} .
$$

We can construct $\boldsymbol{T}=2 \boldsymbol{P}-\boldsymbol{I}$, and the above formula shows that $\boldsymbol{T}$ is nonexpansive. The following lemma shows that the product of two averaged nonexpansive operators is also averaged nonexpansive.

Lemma 3.2. Let $\boldsymbol{P}_{1}$ and $\boldsymbol{P}_{2}$ be $\beta_{1}$ - and $\beta_{2}$-averaged nonexpansive operators, respectively. Then $\boldsymbol{P}_{1} \boldsymbol{P}_{2}$ is $\left(\beta_{1}+\beta_{2}-\beta_{1} \beta_{2}\right)$-averaged nonexpansive.

Proof. Since $\boldsymbol{P}_{1}$ and $\boldsymbol{P}_{2}$ are $\beta_{1}$ - and $\beta_{2}$-averaged nonexpansive operators, there exist nonexpansive operators $\boldsymbol{T}_{1}$ and $\boldsymbol{T}_{2}$ such that

$$
\boldsymbol{P}_{i}=\left(1-\beta_{i}\right) \boldsymbol{I}+\beta_{i} \boldsymbol{T}_{i}
$$

for $i=1,2$. Thus we obtain

$$
\boldsymbol{P}_{1} \boldsymbol{P}_{2}=\left(1-\beta_{1}-\beta_{2}+\beta_{1} \beta_{2}\right) \boldsymbol{I}+\left(1-\beta_{1}\right) \beta_{2} \boldsymbol{T}_{2}+\left(1-\beta_{2}\right) \beta_{1} \boldsymbol{T}_{1}+\beta_{1} \beta_{2} \boldsymbol{T}_{1} \boldsymbol{T}_{2} .
$$

Set $\beta_{3}=\beta_{1}+\beta_{2}-\beta_{1} \beta_{2}$ and

$$
\boldsymbol{T}=\frac{1}{\beta_{1}+\beta_{2}-\beta_{1} \beta_{2}}\left(\left(1-\beta_{1}\right) \beta_{2} \boldsymbol{T}_{2}+\left(1-\beta_{2}\right) \beta_{1} \boldsymbol{T}_{1}+\beta_{1} \beta_{2} \boldsymbol{T}_{1} \boldsymbol{T}_{2}\right) .
$$

It is easy to check that $\beta_{3} \in\left(\beta_{2}, 1\right)$ with $\beta_{1}, \beta_{2} \in(0,1)$ and $\boldsymbol{T}$ is nonexpansive.

Now we show that $\boldsymbol{S}_{h}, \boldsymbol{S}_{w}$, and $\boldsymbol{S}_{t v}$ are firmly nonexpansive. For any $\boldsymbol{x}, \boldsymbol{y} \in \mathbb{R}^{N}$, we have

$$
\boldsymbol{S}_{h}(\boldsymbol{x})-\boldsymbol{S}_{h}(\boldsymbol{y})=\alpha_{1}\left(\boldsymbol{H}^{T} \boldsymbol{H}+\alpha_{1} \boldsymbol{I}\right)^{-1}(\boldsymbol{x}-\boldsymbol{y}) .
$$

It is straightforward to obtain the following inequality:

$$
\begin{aligned}
\left\|\boldsymbol{S}_{h}(\boldsymbol{x})-\boldsymbol{S}_{h}(\boldsymbol{y})\right\|_{2}^{2} & =\left(\boldsymbol{S}_{h}(\boldsymbol{x})-\boldsymbol{S}_{h}(\boldsymbol{y})\right)^{T} \alpha_{1}\left(\boldsymbol{H}^{T} \boldsymbol{H}+\alpha_{1} \boldsymbol{I}\right)^{-1}(\boldsymbol{x}-\boldsymbol{y}) \\
& \leq\left(\boldsymbol{S}_{h}(\boldsymbol{x})-\boldsymbol{S}_{h}(\boldsymbol{y})\right)^{T}(\boldsymbol{x}-\boldsymbol{y}) .
\end{aligned}
$$

It follows that $\boldsymbol{S}_{h}$ is firmly nonexpansive. For $\boldsymbol{S}_{w}$ and $\boldsymbol{S}_{t v}$, we can use the following results in [9]. 
Lemma 3.3 (see [9, Lemma 2.4]). Let $\gamma$ be a positive number and $\varphi$ be a convex and semicontinuous function. Suppose

$$
\widehat{\boldsymbol{x}} \equiv \underset{\boldsymbol{x}}{\operatorname{argmin}}\|\boldsymbol{y}-\boldsymbol{x}\|_{2}^{2}+\gamma \varphi(\boldsymbol{x}),
$$

and define $\boldsymbol{S}: \mathcal{R}^{N} \rightarrow \mathbb{R}^{N}$ such that $\widehat{\boldsymbol{x}}=\boldsymbol{S}(\boldsymbol{y})$. Then $\boldsymbol{S}$ is firmly nonexpansive.

We note that $|x|^{p}(1 \leq p \leq 2)$ is a convex and semicontinuous function. By using the results in Lemma 3.3, both operators $\boldsymbol{S}_{w}$ and $\boldsymbol{S}_{t v}$ are firmly nonexpansive.

In order to establish the convergence of the proposed iterative scheme, we study the following property of $\beta$-averaged nonexpansive operators; see, for instance, [4].

Lemma 3.4. Let $\boldsymbol{P}: \mathbb{R}^{N} \rightarrow \mathbb{R}^{N}$ be a $\beta$-averaged nonexpansive operator. For a given $\boldsymbol{z}^{(0)} \in \mathbb{R}^{N}$, define the sequence $\boldsymbol{z}^{(k)}=\boldsymbol{P}\left(\boldsymbol{z}^{(k-1)}\right)$ for $k=1,2, \ldots$. If the set of fixed points of $\boldsymbol{P}$ is nonempty, then $\boldsymbol{P}$ is asymptotically regular, i.e.,

$$
\lim _{k \rightarrow \infty}\left\|\boldsymbol{z}^{(k+1)}-\boldsymbol{z}^{(k)}\right\|_{2}=\lim _{k \rightarrow \infty}\left\|\boldsymbol{P}^{k+1}\left(\boldsymbol{z}^{(0)}\right)-\boldsymbol{P}^{k}\left(\boldsymbol{z}^{(0)}\right)\right\|_{2}=0 .
$$

Proof. Let $\boldsymbol{z}$ be a fixed point of $\boldsymbol{P}$. We denote $\boldsymbol{g}=\boldsymbol{I}-\boldsymbol{P}$. Since $\boldsymbol{P}$ is a $\beta$-averaged nonexpansive operator, there exist a number $\beta \in(0,1)$ and a nonexpansive operator $\boldsymbol{T}$ such that $\boldsymbol{P}=(1-\beta) \boldsymbol{I}+\beta \boldsymbol{T}$. It also implies that $\boldsymbol{g}=\beta(\boldsymbol{I}-\boldsymbol{T})$. For any $\boldsymbol{x}, \boldsymbol{y} \in \mathbb{R}^{N}$, we have

$$
\|\boldsymbol{x}-\boldsymbol{y}\|_{2}^{2}-\|\boldsymbol{T}(\boldsymbol{x})-\boldsymbol{T}(\boldsymbol{y})\|_{2}^{2}=\frac{2}{\beta}(\boldsymbol{g}(\boldsymbol{x})-\boldsymbol{g}(\boldsymbol{y}))^{T}(\boldsymbol{x}-\boldsymbol{y})-\frac{1}{\beta^{2}}\|\boldsymbol{g}(\boldsymbol{x})-\boldsymbol{g}(\boldsymbol{y})\|_{2}^{2} \geq 0
$$

or

$$
[\boldsymbol{g}(\boldsymbol{x})-\boldsymbol{g}(\boldsymbol{y})]^{T}(\boldsymbol{x}-\boldsymbol{y}) \geq \frac{1}{2 \beta}\|\boldsymbol{g}(\boldsymbol{x})-\boldsymbol{g}(\boldsymbol{y})\|_{2}^{2} .
$$

By using the fact that $\boldsymbol{z}=\boldsymbol{P}(\boldsymbol{z})$, we have

$$
\begin{aligned}
\left\|\boldsymbol{z}-\boldsymbol{z}^{(k)}\right\|_{2}^{2}-\left\|\boldsymbol{z}-\boldsymbol{z}^{(k+1)}\right\|_{2}^{2} & =\left\|\boldsymbol{z}-\boldsymbol{z}^{(k)}\right\|_{2}^{2}-\left\|\boldsymbol{P}(\boldsymbol{z})-\boldsymbol{P}\left(\boldsymbol{z}^{(k)}\right)\right\|_{2}^{2} \\
& =2\left(\boldsymbol{g}(\boldsymbol{z})-\boldsymbol{g}\left(\boldsymbol{z}^{(k)}\right)\right)^{T}\left(\boldsymbol{z}-\boldsymbol{z}^{(k)}\right)-\left\|\boldsymbol{g}(\boldsymbol{z})-\boldsymbol{g}\left(\boldsymbol{z}^{(k)}\right)\right\|_{2}^{2} \\
& \geq\left(\frac{1}{\beta}-1\right)\left\|\boldsymbol{g}(\boldsymbol{z})-\boldsymbol{g}\left(\boldsymbol{z}^{(k)}\right)\right\|_{2}^{2}
\end{aligned}
$$

We note that $\boldsymbol{g}(\boldsymbol{z})=\boldsymbol{z}-\boldsymbol{P}(\boldsymbol{z})=0$ and $\boldsymbol{g}\left(\boldsymbol{z}^{(k)}\right)=\boldsymbol{z}^{(k)}-\boldsymbol{z}^{(k+1)}$. Therefore, we obtain

$$
\left\|\boldsymbol{z}-\boldsymbol{z}^{(k)}\right\|_{2}^{2}-\left\|\boldsymbol{z}-\boldsymbol{z}^{(k+1)}\right\|_{2}^{2} \geq\left(\frac{1}{\beta}-1\right)\left\|\boldsymbol{z}^{(k)}-\boldsymbol{z}^{(k+1)}\right\|_{2}^{2} .
$$

Since $(1 / \beta-1)>0$, one can immediately deduce that $\sum_{k}\left\|\boldsymbol{z}^{(k)}-\boldsymbol{z}^{(k+1)}\right\|_{2}^{2}$ is bounded. Then the result follows.

Now we consider the well-known Opial theorem [27]. It states that if the set of fixed points of $\boldsymbol{P}$ is nonempty, and $\boldsymbol{P}$ is nonexpansive and asymptotically regular, then the sequence $\boldsymbol{x}^{(k)}$, where $\boldsymbol{x}^{(k)}=\boldsymbol{P}\left(\boldsymbol{x}^{(k-1)}\right)$ for $k=1,2, \ldots$, converges weakly to a fixed point. Using the results in Lemma 3.4, we have the following theorem.

Theorem 3.5 (see [4, Theorem 2.1]). Let $\boldsymbol{P}: \mathbb{R}^{N} \rightarrow \mathbb{R}^{N}$ be a $\beta$-averaged nonexpansive operator. If the set of fixed points of $\boldsymbol{P}$ is nonempty, then for any $\boldsymbol{z}^{(0)}$, the sequence $\left\{\boldsymbol{z}^{(k)}\right\}$, where $\boldsymbol{z}^{(k)}=\boldsymbol{P}\left(\boldsymbol{z}^{(k-1)}\right)$ for $k=1,2, \ldots$, converges to a fixed point in $\mathbb{R}^{N}$. 
According to Lemma 3.2, since the operators $\boldsymbol{S}_{h}, \boldsymbol{S}_{w}$, and $\boldsymbol{S}_{t v}$ are firmly nonexpansive, the product $\boldsymbol{S}_{t v} \boldsymbol{S}_{w} \boldsymbol{S}_{h}$ is $\beta$-averaged nonexpansive for some $\beta \in(0,1)$. By applying Theorem 3.5, we have our main results of this paper.

TheOREm 3.6. (i) Assuming that the set of fixed points of $\boldsymbol{S}_{w} \boldsymbol{S}_{h}$ is nonempty, Algorithm 1 converges to a fixed point.

(ii) Assuming that the set of fixed points of $\boldsymbol{S}_{t v} \boldsymbol{S}_{h}$ is nonempty, Algorithm 2 converges to a fixed point.

(iii) Assuming that the set of fixed points of $\boldsymbol{S}_{t v} \boldsymbol{S}_{w} \boldsymbol{S}_{h}$ is nonempty, Algorithm 3 converges to a fixed point.

Before we end this section, we give several remarks about the convergence of the proposed algorithm.

- When $\boldsymbol{H}$ is rank deficient, $\boldsymbol{S}_{w} \boldsymbol{S}_{h}, \boldsymbol{S}_{t v} \boldsymbol{S}_{h}$, and $\boldsymbol{S}_{t v} \boldsymbol{S}_{w} \boldsymbol{S}_{h}$ may not strictly be contraction operators. However, we can add a projection operator $\boldsymbol{S}_{p}$ at each iteration such that the values of the entries of $\boldsymbol{f}^{(i+1)}$ are projected to $[0,255]$. It follows from the Banach-Algoglu theorem that the operators $\boldsymbol{S}_{p} \boldsymbol{S}_{w} \boldsymbol{S}_{h}, \boldsymbol{S}_{p} \boldsymbol{S}_{t v} \boldsymbol{S}_{h}$, and $\boldsymbol{S}_{p} \boldsymbol{S}_{t v} \boldsymbol{S}_{w} \boldsymbol{S}_{h}$ have a weak accumulation point; see [10] for details. Therefore, their corresponding sets of fixed points are nonempty. By using Theorem 3.6, we can show that Algorithms 1,2, and 3 converge to a fixed point.

- When $\boldsymbol{H}$ is full rank, i.e., the smallest eigenvalue of $\boldsymbol{H}^{T} \boldsymbol{H}$ is larger than 0, it is easy to check that, for all $\boldsymbol{x}, \boldsymbol{y}$, there exists a number $r \in(0,1)$ such that

$$
\left\|\boldsymbol{S}_{h}(\boldsymbol{x})-\boldsymbol{S}_{h}(\boldsymbol{y})\right\|_{2} \leq r\|\boldsymbol{x}-\boldsymbol{y}\|_{2} .
$$

It implies that $\boldsymbol{S}_{w} \boldsymbol{S}_{h}, \boldsymbol{S}_{t v} \boldsymbol{S}_{h}$, and $\boldsymbol{S}_{t v} \boldsymbol{S}_{w} \boldsymbol{S}_{h}$ are strictly contraction operators. According to the Banach-Picard theorem, for any initial vector $\boldsymbol{f}^{(0)}$, Algorithms 1, 2, and 3 converge to a unique fixed point.

4. Numerical results. In this section, we illustrate the performance of Algorithms 1, 2, and 3 for solving image restoration problems. Three indices are used to measure the quality of the restored images by different algorithms. They are the signal-to-noise ratio (SNR), the improvement in SNR (ISNR), and the blurred SNR (BSNR):

$$
\mathrm{SNR}=10 \log _{10} \frac{\|\boldsymbol{f}\|_{2}^{2}}{\|\boldsymbol{f}-\widetilde{\boldsymbol{f}}\|_{2}^{2}}, \quad \text { ISNR }=10 \log _{10} \frac{\|\boldsymbol{f}-\boldsymbol{g}\|_{2}^{2}}{\|\boldsymbol{f}-\widetilde{\boldsymbol{f}}\|_{2}^{2}}
$$

and

$$
\mathrm{BSNR}=10 \log _{10} \frac{\|\boldsymbol{g}\|_{2}^{2}}{\|\boldsymbol{n}\|_{2}^{2}}
$$

where $\boldsymbol{f}, \boldsymbol{g}, \widetilde{\boldsymbol{f}}$, and $\boldsymbol{n}$ are the original image, the blurred and noisy image, the restored image, and the noise vector, respectively. In our algorithms, the stopping criterion is

$$
\frac{\left\|\boldsymbol{f}^{(k-1)}-\boldsymbol{f}^{(k)}\right\|_{2}}{\left\|\boldsymbol{f}^{(k-1)}\right\|_{2}} \leq 10^{-4} .
$$

Copyright (c) by SIAM. Unauthorized reproduction of this article is prohibited. 
We use the output of the ForWaRD restoration method [23] as an initial image. Trial by error for $\alpha_{1}$ and $\alpha_{2}$ is used in the tests. In the figures, we report the highest SNR of the restored image over all tested values of $\alpha_{1}$. The corresponding values of $\alpha_{1}$ are reported in the figures. In all the tests, $\alpha_{2}$ is fixed and set to be 0.8 .

Similar to [13, 23], we employ the Haar wavelet shrinkage denoising methods. The shrinkage methods include the soft thresholding method [12], the hard thresholding method [22], and the garrote thresholding method [16]. The program for the TV denoising method is based on the code ${ }^{1}$ written by Vogel [33]. A very small parameter is set in the TV term to avoid singularities in the calculation in all the tests; for details see [33].

In the first experiment, we use a synthetic $128 \times 128$ image as shown as in Figure 5 . The image is blurred by a $9 \times 9$ point box-car blur $[1,23]$ defined as follows:

$$
h(i, j)= \begin{cases}\frac{1}{81}, & 0 \leq i, j \leq 8 \\ 0 & \text { otherwise }\end{cases}
$$

White Gaussian noises are added to the blurred image. The blurred and noisy images are shown as in Figures 6(left) and 6(right) for BSNR $=20 \mathrm{~dB}$ and BSNR $=30 \mathrm{~dB}$, respectively.

Figures 7 and 11 show the restored images by using ForWaRD [23], the wavelet regularization in (1.2) [13], and the TV regularization in (1.2) for the restoration of blurred noisy images with BSNR $=20 \mathrm{~dB}$ and BSNR $=30 \mathrm{~dB}$, respectively. Figures 8 and 12 show the restored images by using Algorithm 1 with hard thresholding, soft thresholding, and garrote thresholding for the restoration of blurred noisy images with $\mathrm{BSNR}=20 \mathrm{~dB}$ and BSNR $=30 \mathrm{~dB}$, respectively. Figures 9 and 13 show the restored image by using Algorithm 2 for the blurred noisy images with BSNR $=20 \mathrm{~dB}$ and $\mathrm{BSNR}=30 \mathrm{~dB}$, respectively. Figures 10 and 14 show the restored images by using Algorithm 3 with hard thresholding, soft thresholding, and garrote thresholding for the restoration of blurred noisy images with with BSNR $=20 \mathrm{~dB}$ and BSNR $=30 \mathrm{~dB}$, respectively. We find in Figures $8-10$ and $12-14$ that the proposed algorithms can produce better restored images in visual quality and SNR than those by the restoration methods using the combination of a data-fitting term and a regularization term.

Next we discuss the convergence of Algorithms 1, 2, and 3. Figures 15-17 give the convergence curves of the three algorithms corresponding to Figures 8-10. Figures 1820 give the convergence curves of the three algorithms corresponding to Figures 12-14. Here the $y$-axis refers to successive errors of the iterates: $\frac{\left\|\boldsymbol{f}^{(k-1)}-\boldsymbol{f}^{(k)}\right\|_{2}}{\left\|\boldsymbol{f}^{(k-1)}\right\|_{2}}$. We find that the three proposed algorithms take about 15-25 iterations such that the successive errors of the iterates are less than $10^{-4}$ (the stopping criterion). We also see that the convergence rate is very quick in the first few iterations, and then it becomes slower, especially when the successive errors are close to the stopping criterion.

In the second experiment, we test the proposed algorithms and compare with other restoration methods using several different blurs studied in [13]:

$$
\begin{aligned}
& h(i, j)= \begin{cases}\frac{1}{1+i^{2}+j^{2}}, & -7 \leq i, j \leq 7, \\
0 & \text { otherwise; }\end{cases} \\
& h(i, j)=[1,4,6,4,1]^{T}[1,4,6,4,1] / 256 .
\end{aligned}
$$

\footnotetext{
${ }^{1} \mathrm{http}: / /$ www.math.montana.edu/ vogel/Book/
} 
Original Image

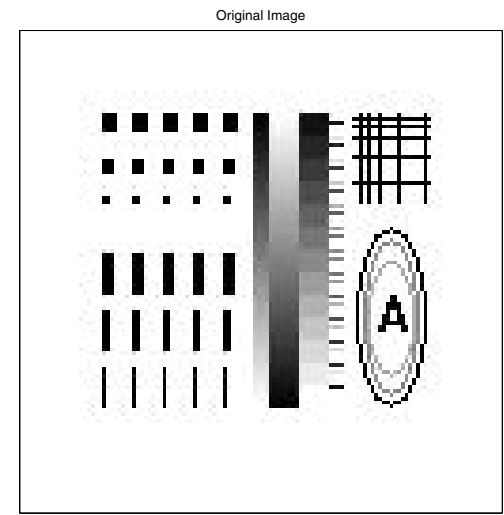

FIG. 5. The original image.
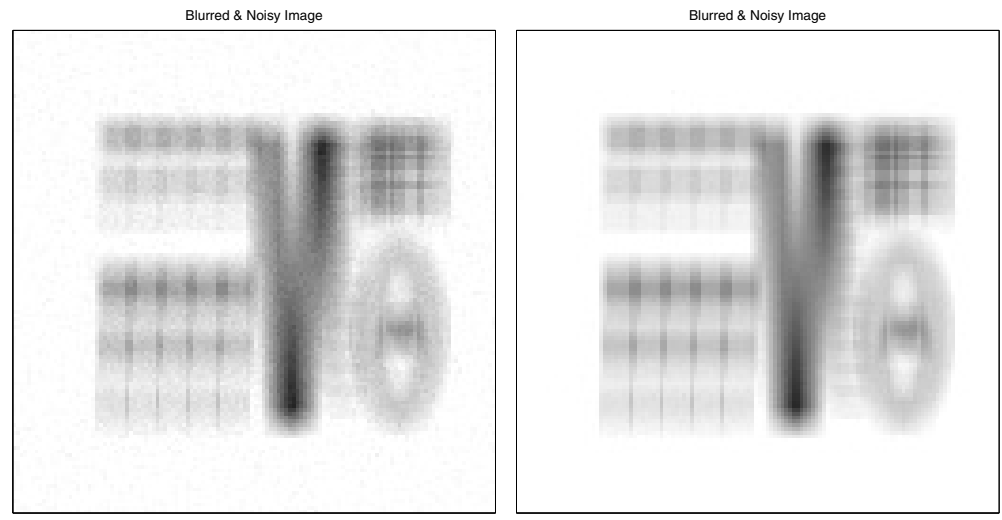

FIG. 6. The blurred and noisy images for $B S N R=20 \mathrm{~dB}$ (left) and $B S N R=30 \mathrm{~dB}$ (right). 
ForWaRD, SNR:18.17 a:0.00098971

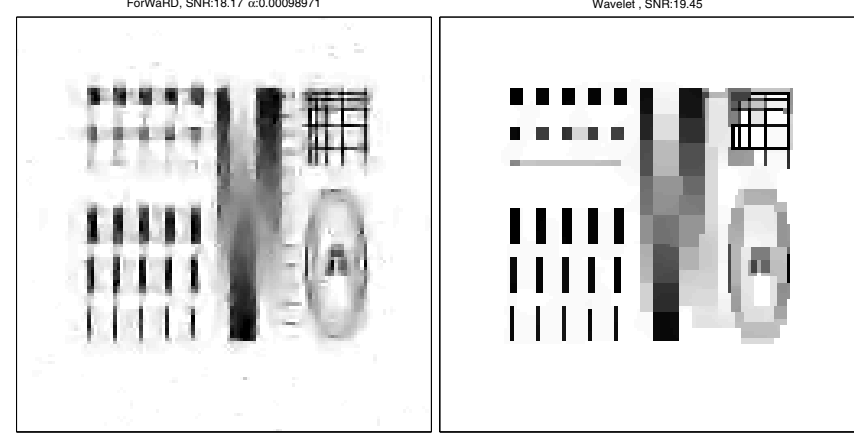

TV, SNR:18.98 a:0.19789

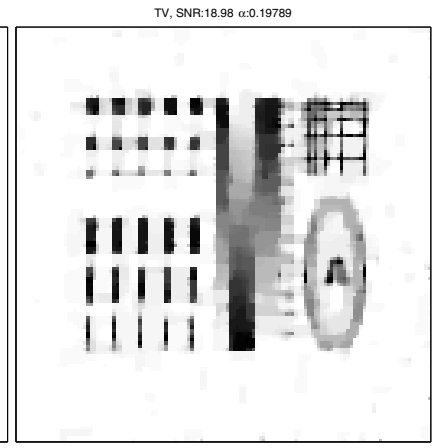

FIG. 7. The restored images by using For WaRD [23] [SNR $=18.17 \mathrm{~dB}$ ] (left), wavelet regularization in $(1.2)$ [13] [SNR $=19.45 \mathrm{~dB}$ ] (middle), and TV regularization in 1.2 [SNR $=18.98 \mathrm{~dB}$ ] (right).

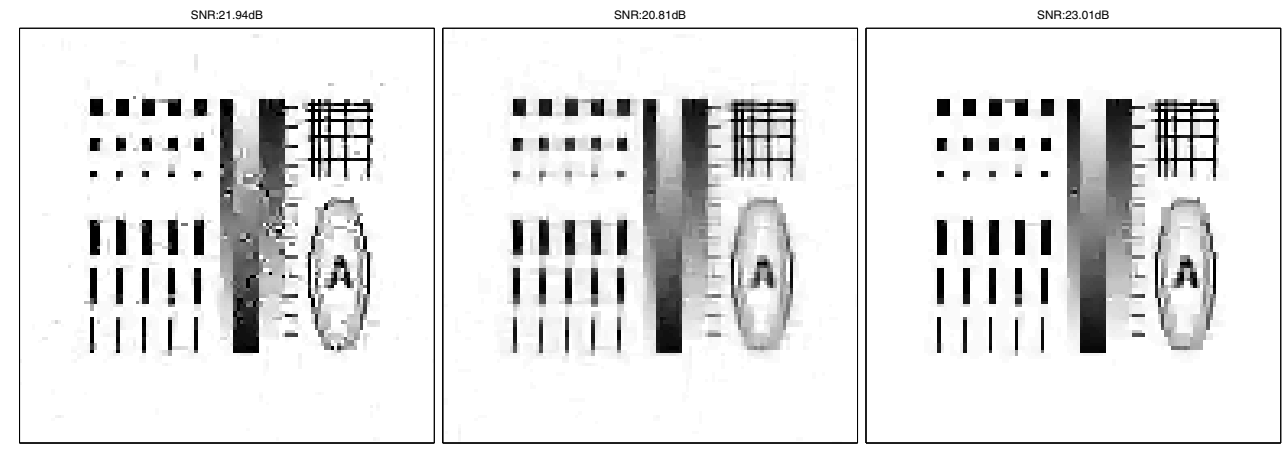

FIG. 8. The restored images by using Algorithm 1 with hard thresholding $\left(\alpha_{1}=0.018\right)$ [SNR $=$ $21.94 \mathrm{~dB}]$ (left), soft thresholding $\left(\alpha_{1}=0.06\right)$ [SNR $\left.=20.81 \mathrm{~dB}\right]$ (middle), and garrote shrinkage $\left(\alpha_{1}=0.06\right)[S N R=23.01 \mathrm{~dB}]$ (right).

Copyright $@$ by SIAM. Unauthorized reproduction of this article is prohibited. 


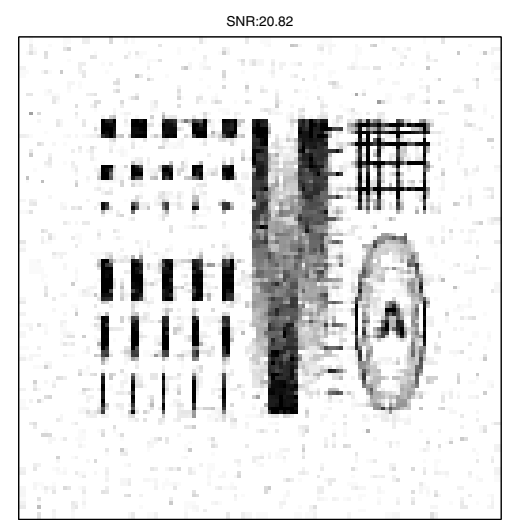

FIG. 9. The restored image by using Algorithm $2\left(\alpha_{1}=0.06\right)$ [SNR $\left.=20.82 d B\right]$.

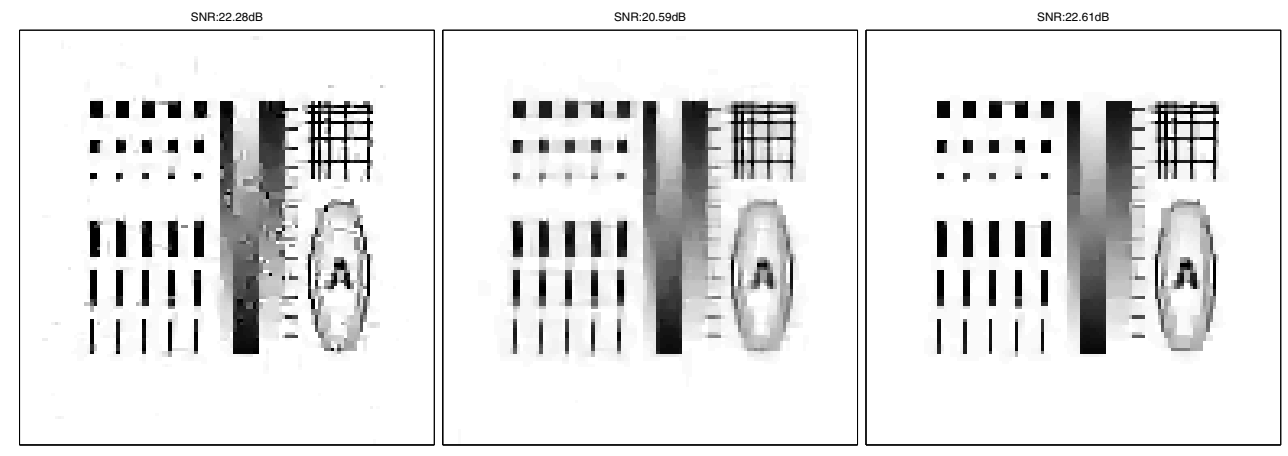

FIG. 10. The restored images by using Algorithm 3 with hard thresholding $\left(\alpha_{1}=0.018\right)$ [SNR $=$ $22.28 \mathrm{~dB}]$ (left), soft thresholding $\left(\alpha_{1}=0.06\right)$ [SNR $\left.=20.59 \mathrm{~dB}\right]$ (middle), and garrote shrinkage $\left(\alpha_{1}=0.06\right)[S N R=22.61 \mathrm{~dB}]$ (right). 


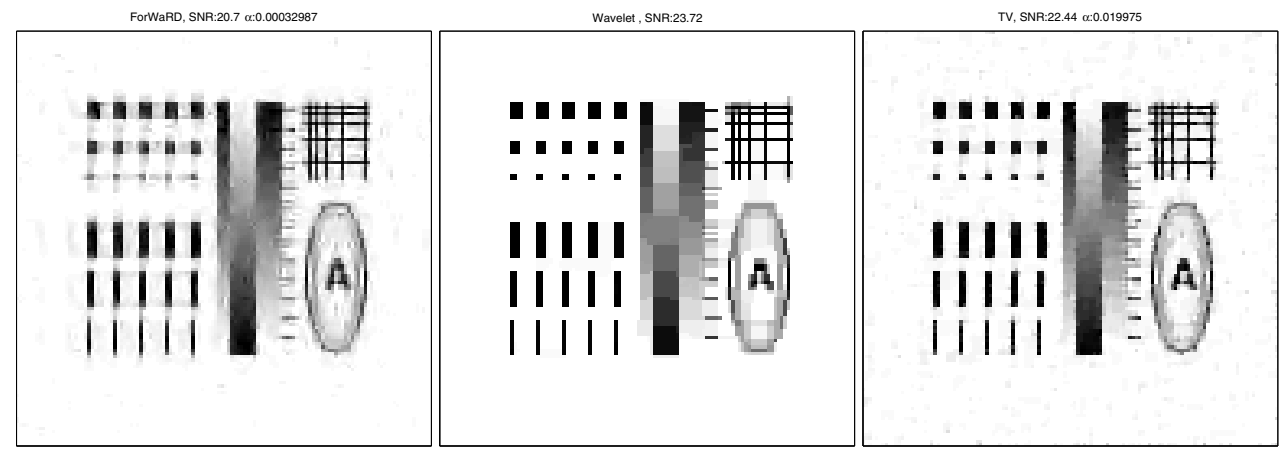

FIG. 11. The restored images by using For WaRD [23] [SNR $=20.70 \mathrm{~dB}$ ] (left), wavelet regularization in (1.2) [13] [SNR $=23.72 \mathrm{~dB}]$ (middle), and TV regularization in 1.2 [SNR $=22.44 \mathrm{~dB}$ ] (right).

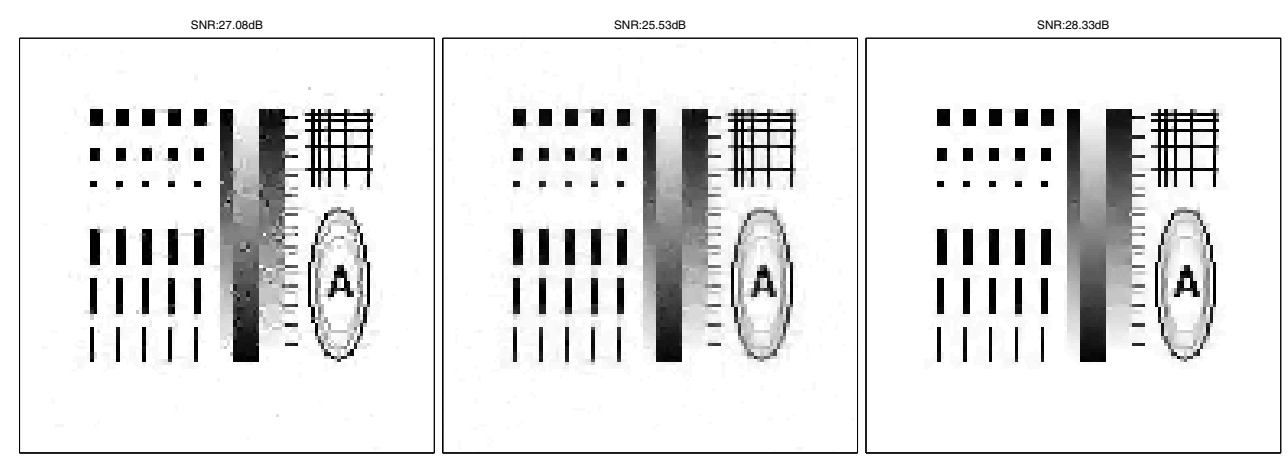

FIG. 12. The restored images by using Algorithm 1 with hard thresholding $\left(\alpha_{1}=0.005\right)$ [SNR $=$ $27.08 \mathrm{~dB}$ ] (left), soft thresholding $\left(\alpha_{1}=0.005\right)[S N R=25.53 \mathrm{~dB}]$ (middle), and garrote shrinkage $\left(\alpha_{1}=0.005\right)[S N R=28.33 \mathrm{~dB}]$ (right).

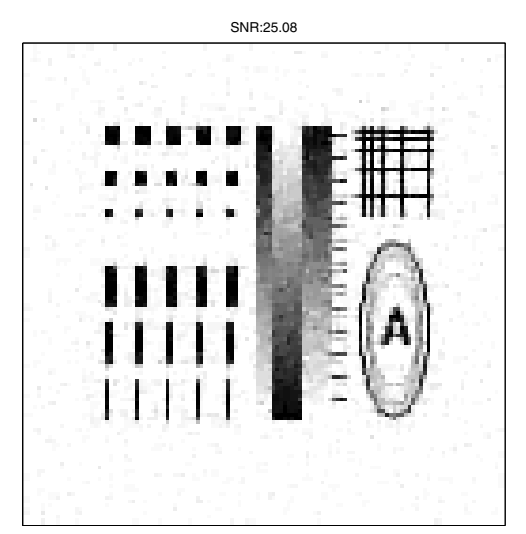

FIG. 13. The restored image by using Algorithm $2\left(\alpha_{1}=0.02\right)$ [SNR $\left.=25.08 d B\right]$. 


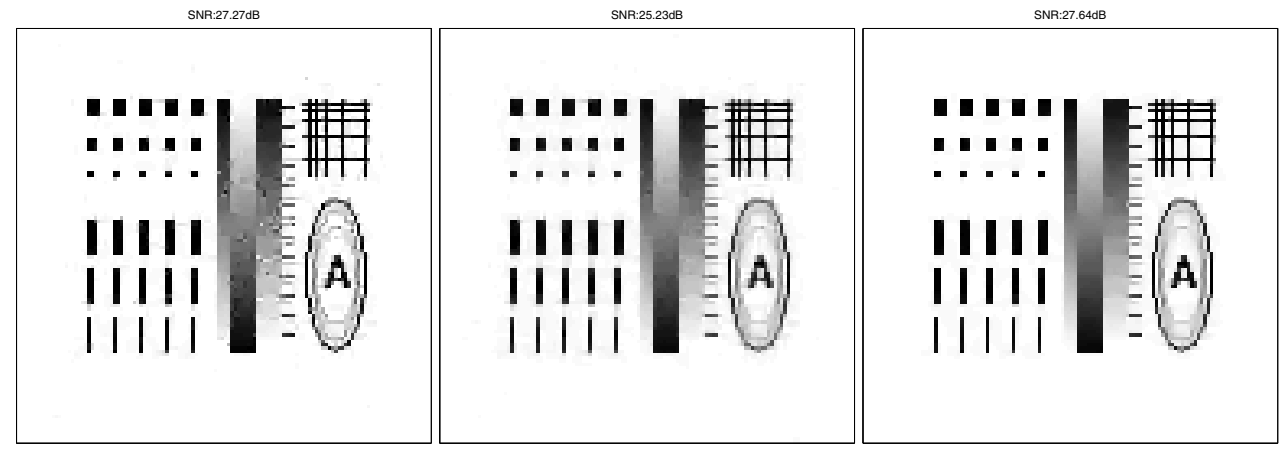

FIG. 14. The restored images by using Algorithm 3 with hard thresholding $\left(\alpha_{1}=0.005\right)$ [SNR $=$ $27.27 \mathrm{~dB}$ ] (left), soft thresholding $\left(\alpha_{1}=0.005\right)$ [SNR $\left.=25.23 \mathrm{~dB}\right]$ (middle), and garrote shrinkage $\left(\alpha_{1}=0.005\right)[S N R=27.64 d B]$ (right).
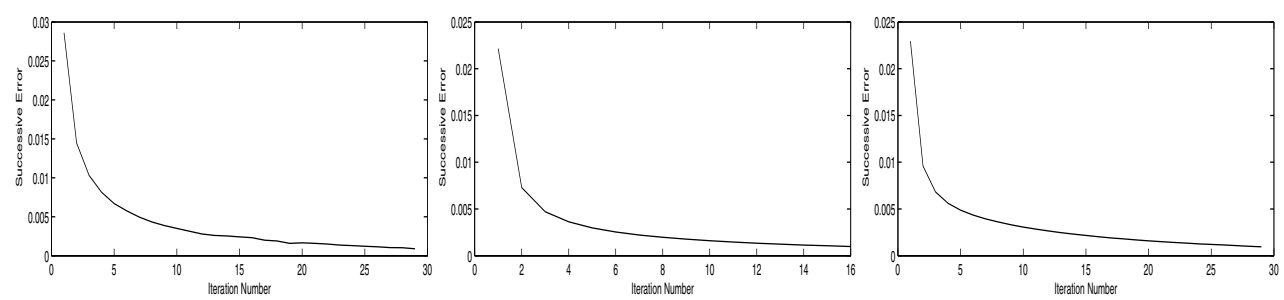

FIG. 15. The convergence curves of Algorithm 1 with hard thresholding (left), soft thresholding (middle), and garrote shrinkage (right).

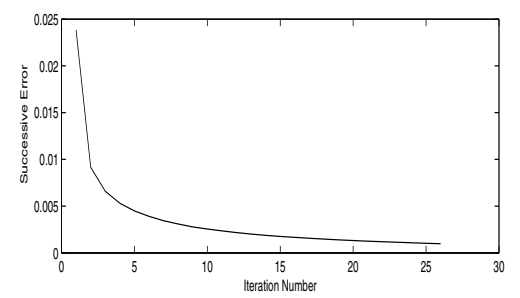

FIG. 16. The convergence curves of Algorithm 2.
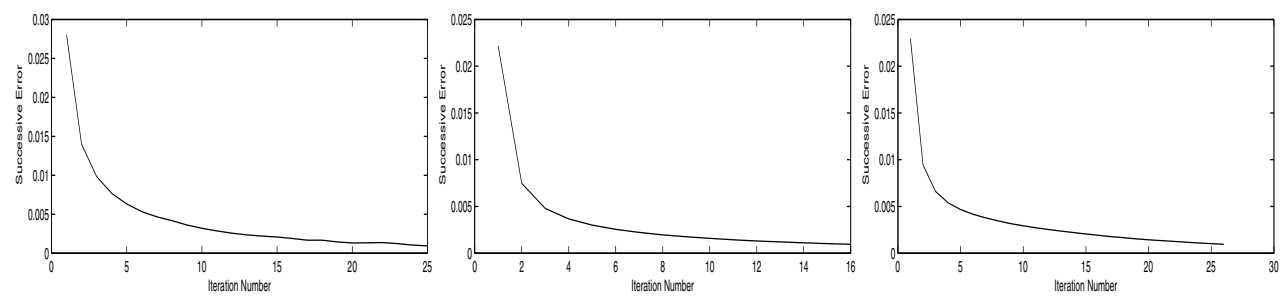

FIG. 17. The convergence curves of Algorithm 3 with hard thresholding (left), soft thresholding (middle), and garrote shrinkage (right).

Copyright $@$ ㅇ by SIAM. Unauthorized reproduction of this article is prohibited. 

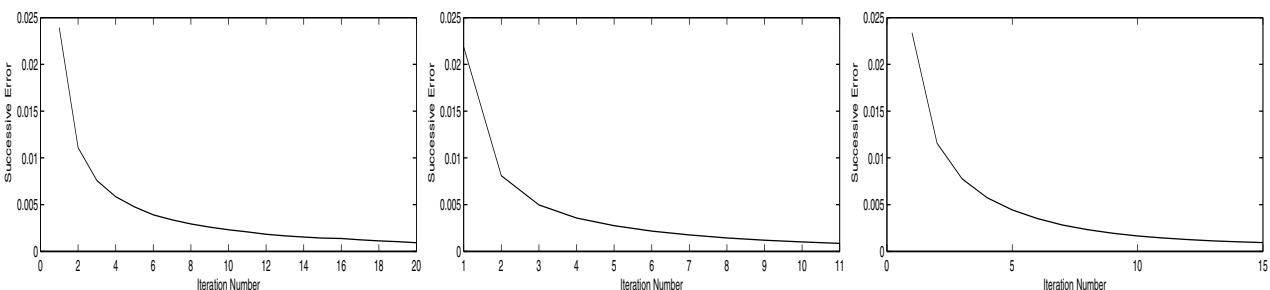

FIG. 18. The convergence curves of Algorithm 1 with hard thresholding (left), soft thresholding (middle), and garrote shrinkage (right).

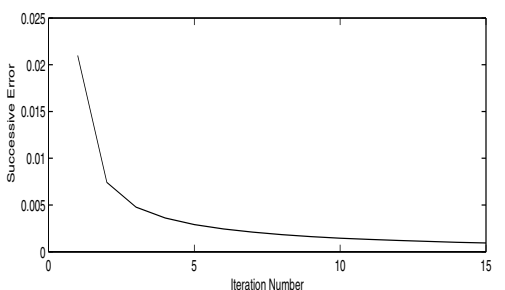

FIG. 19. The convergence curves of Algorithm 2.
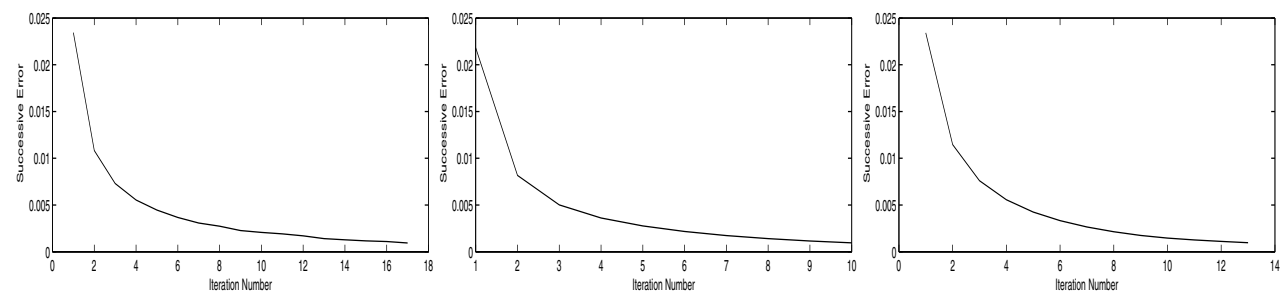

FIG. 20. The convergence curves of Algorithm 3 with hard thresholding (left), soft thresholding (middle), and garrote shrinkage (right).

In the tests, we set the variances $\sigma$ of noises that have been used in the published papers $[1,2,13,14,19,20,21,23]$. The detailed information about the tests is given in Table 1. There are several image restoration methods $[1,2,13,14,19,20,21,23]$ used for comparison. Their restoration results in their published work under the same setting are listed in Table 2. In particular, we show the ISNRs of the proposed algorithms and other restoration methods. The symbol "-" in the table indicates that the settings are not tested in the corresponding published work. According to Table 2 , we observe that the performances of our proposed algorithms are quite good and are comparable to the other restoration methods in the literature.

5. Concluding remarks. We have proposed iterative algorithms for solving image restoration problems. The iterative algorithms are based on decoupling of deblurring and denoising steps in the restoration process. The main advantage of this proposal is that the resulting algorithms can be very efficient and can produce better restored images in visual quality and SNR than those by the restoration methods 
TABLE 1

The blurs, variances of noises, and original images.

\begin{tabular}{l|c|c|c|c}
\hline & Blur & Noise variance & Image & Size \\
\hline Example 1 & blur in (4.1) & 0.31 & cameraman & $256 \times 256$ \\
Example 2 & blur in (4.2) & 2 & cameraman & $256 \times 256$ \\
Example 3 & blur in (4.2) & 8 & cameraman & $256 \times 256$ \\
Example 4 & blur in (4.3) & 49 & Lena & $256 \times 256$ \\
\hline
\end{tabular}

TABLE 2

SNR Improvement in different examples.

\begin{tabular}{l|c|c|c|c}
\hline Method & Ex. 1 & Ex. 2 & Ex. 3 & Ex. 4 \\
\hline Algorithm 1 with hard thresholding & 8.46 & 7.53 & 5.64 & 2.90 \\
$\alpha_{1}$ & 0.05 & 0.50 & 1.40 & 8.00 \\
\hline Algorithm 1 with soft thresholding & 8.57 & 7.53 & 5.56 & 2.81 \\
$\alpha_{1}$ & 0.02 & 0.10 & 0.40 & 1.60 \\
\hline Algorithm 1 with garrote thresholding & 8.77 & 7.58 & 5.69 & 2.90 \\
$\alpha_{1}$ & 0.06 & 0.40 & 1.40 & 4.80 \\
\hline Algorithm 2 & 8.81 & 7.52 & 5.56 & 2.58 \\
$\alpha_{1}$ & 0.05 & 0.30 & 1.00 & 2.50 \\
\hline Algorithm 3 with hard thresholding & 8.91 & 7.74 & 5.69 & 3.04 \\
$\alpha_{1}$ & 0.006 & 0.10 & 0.27 & 1.60 \\
\hline Algorithm 3 with soft thresholding & 8.51 & 7.39 & 5.46 & 2.74 \\
$\alpha_{1}$ & 0.006 & 0.04 & 0.13 & 0.50 \\
\hline Algorithm 3 with garrote thresholding & 8.87 & 7.60 & 5.69 & 2.91 \\
$\alpha_{1}$ & 0.006 & 0.10 & 0.27 & 0.80 \\
\hline TV [3] & 8.41 & - & - & 2.80 \\
ForWaRD [23] & 7.34 & 6.72 & 4.95 & 2.26 \\
EM [13] & 7.59 & 6.93 & 4.88 & 2.94 \\
BOA [14] & 8.16 & 7.46 & 5.24 & 2.84 \\
GEM [2] & 8.10 & 7.40 & 5.15 & 2.85 \\
Banham \& Katsaggelos [1] & 6.70 & - & - & - \\
Jalobeanu et al. [19] & - & 6.75 & 4.85 & - \\
Liu \& Moulin [21] & - & - & - & 1.08 \\
RI-RWI [20] & 7.84 & 7.31 & 5.54 & - \\
\hline
\end{tabular}

using the combination of a data-fitting term and a regularization term. Numerical results have shown that the proposed algorithms are quite effective.

Acknowledgment. The authors would like to thank the two reviewers for their suggestions that led to an improved presentation of the paper.

\section{REFERENCES}

[1] M. Banham And A. Katsaggelos, Spatially adaptive wavelet-based multiscale image restoration, IEEE Trans. Image Process., 5 (1996), pp. 619-634.

[2] J. Bioucas-Dias, Bayesian wavelet-based image deconvolution: A GEM algorithm exploiting a class of heavy-tailed priors, IEEE Trans. Image Process., 15 (2006), pp. 937-951.

[3] J. Bioucas-Dias, M. Figueiredo, and R. Nowak, Total variation-based image deconvolution: A majorization-minimization approach, in Proceedings of the IEEE International Conference on Acoustics, Speech, and Signal Processing (ICASSP 2006), Toulouse, France, 2006.

[4] C. BYRne, A unified treatment of some iterative algorithms in signal processing and image reconstruction, Inverse Problems, 20 (2004), pp. 103-120.

[5] A. Chambolle, An algorithm for total variation minimization and applications, J. Math. Imaging Vision, 20 (2004), pp. 89-97.

[6] T. F. Chan And K. Chen, An optimization-based multilevel algorithm for total variation image denoising, Multiscale Model. Simul., 5 (2006), pp. 615-645. 
[7] T. F. Chan and P. Mulet, On the convergence of the lagged diffusivity fixed point method in total variation image restoration, SIAM J. Numer. Anal., 36 (1999), pp. 354-367.

[8] P. L. Combettes, Solving monotone inclusions via compositions of nonexpansive averaged operators, Optimization, 53 (2004), pp. 475-504.

[9] P. L. Combettes And V. R. Wajs, Signal recovery by proximal forward-backward splitting, Multiscale Model. Simul., 4 (2005), pp. 1168-1200.

[10] I. Daubechies, M. Defrise, And C. De Mol, An iterative thresholding algorithm for linear inverse problems with a sparsity constraint, Comm. Pure Appl. Math., 57 (2004), pp. 14131457.

[11] D. Donoho, Nonlinear solution of linear inverse problems by wavelet-vaguelette decompositions, Appl. Comput. Harmon. Anal., 1 (1995), pp. 100-115.

[12] D. DonoHo, De-noising by soft-thresholding, IEEE Trans. Inform. Theory, 41 (1995), pp. 613627.

[13] M. FigueIRedo And R. Nowak, An EM algorithm for wavelet-based image restoration, IEEE Trans. Image Process., 12 (2003), pp. 906-916.

[14] M. Figueiredo AND R. NowAK, A bound optimization approach to wavelet-based image deconvolution, in Proceedings of the IEEE International Conference on Image Processing (ICIP 2005), Genoa, Italy, 2005.

[15] N. Galatsanos and A. Katsaggelos, Methods for choosing the regularization parameter and estimating the noise variance in image restoration and their relation, IEEE Trans. Image Process., 1 (1992), pp. 322-336.

[16] H. GaO, Wavelet shrinkage denoising using the non-negative garrote, J. Comput. Graph. Statist., 7 (1998), pp. 469-488.

[17] R. Gonzalez and P. Wintz, Digital Image Processing, Addison-Wesley, Reading, MA, 1977.

[18] R. Gopinath, M. Lang, H. Guo, and J. Odegard, Enhancement of decompressed images at low bit rates, in Mathematical Imaging: Wavelet Applications in Signal And Image Processing, Vol. 2303, SPIE, Bellingham, WA, 1994, pp. 366-377.

[19] A. Jalobeanu, N. Kingsbury, and J. Zerubia, Image deconvolution using hidden Markov tree modeling of complex wavelet packets, in Proceedings of the IEEE International Conference Image Processing, Thessaloniki, Greece, 2001.

[20] V. Katkovnik, K. Egiazarian, and J. Astola, A spatially adaptive nonparametric regression image deblurring, IEEE Trans. Image Process., 14 (2005), pp. 1469-1478.

[21] J. Liu And P. Moulin, Complexity regularized image restoration, in Proceedings of the IEEE International Conference on Image Processing (ICIP '98), Vol. 1, 1998, pp. 555-559.

[22] S. Mallat, A Wavelet Tour of Signal Processing, 2nd ed., Academic Press, San Diego, 1999.

[23] R. Neelamani, H. Choi, and R. Baraniuk, ForWaRD: Fourier-wavelet regularized deconvolution for ill-conditioned systems, IEEE Trans. Signal Process., 52 (2004), pp. 418-433.

[24] M. NG, Iterative Methods for Toeplitz Systems, Oxford University Press, Oxford, UK, 2004.

[25] M. K. NG, R. H. Chan, And W.-C. TANG, A fast algorithm for deblurring models with Neumann boundary conditions, SIAM J. Sci. Comput., 21 (1999), pp. 851-866.

[26] M. NG, L. QI, Y. YAng, AND Y. HuAng, On semismooth Newton's methods for total variation minimization, J. Math. Imaging Vision, 27 (2007), pp. 265-276.

[27] Z. OpIAL, Weak convergence of the sequence of successive approximations for nonexpansive mappings, Bull. Amer. Math. Soc., 73 (1967), pp. 591-597.

[28] N. Paragios, C. Chen, and O. Faugeras, eds., Handbook of Mathematical Models in Computer Vision, Springer, New York, 2006.

[29] L. I. Rudin, S. OSher, AND E. FAtemi, Nonlinear total variation based noise removal algorithms, Phys. D, 60 (1992), pp. 259-268.

[30] G. Steidl, J. Weickert, T. A. Brox, P. Mrázek, and M. Welk, On the equivalence of soft wavelet shrinkage, total variation diffusion, total variation regularization, and SIDEs, SIAM J. Numer. Anal., 42 (2004), pp. 686-713.

[31] D. Terzopoulos, Regularization of inverse visual problems involving discontinuities, IEEE Trans. Pattern Anal. Mach. Intell., 8 (1986), pp. 413-424.

[32] A. TikHonov, Solution of incorrectly formulated problems and the regularization method, Soviet Math. Dokl., 4 (1963), pp. 1035-1038.

[33] C. R. Vogel, Computational Methods for Inverse Problems, SIAM, Philadelphia, 2002.

[34] J. B. Weaver, Y. Xu, D. M. Healy, and L. D. Cromwell, Filtering noise from images with wavelet transforms, Magn. Reson. Med., 21 (1991), pp. 288-295.

Copyright $@$ by SIAM. Unauthorized reproduction of this article is prohibited. 